\title{
A Solution for the Problem of Interactive Disambiguation
}

\author{
Hervé BLANCHON
}

\author{
GETA, Institut IMAG (UJF \& CNRS) \\ HP 53X, 38041 Grenoble Cedex, France \\ e-mail : blanclıon@ imag.fr
}

\section{Extended abstract}

After the experiences of dialogue based MT systems with ITS [9], N.Tran [6] and KBMT-89 [5], the LIDIA project aims at the construction of a mock-up of a personal MT system for a momolingual user. One major aspect of the I.IDIA project is thus, the study of a dialogue of standardization and disambiguation between the system and the user so as to produce a high quality translation. This dialogue satisfies two properties: its questions are explicit, so no linguistic knowledge is required; its questions are monolingual, so no foreign language knowledge is needed. Here, we focus on one part of the disambiguation process: the disambiguation of the structure produced by the analyser.

The structure produced by our analyser is called MMC (Multisolution, Multilevel and Concrete). Multisolution means that the analyser produces every analysis fitting with the syntagmatic, syntactic and logico-semantic model of the grammar (an example is shown fig. 1). Multilevel means that the same structure consists of three levels of linguistic interpretation, namely the level of syntactic and syntagmatic classes, the level of syntactic functions and the level of logic and semantic relations. Finally, the structure is said to be concrete because the original utterance can be found back by a simple left-to-right reading of the structure.

We have taken into account three kinds of differences between the solutions produced for one sentence, and each kind of difference is associated with the name of an ambiguity. We have defined ambiguities of syntactic classes (cf fig. 2), ambiguities of geometry (cf fig. 3) and ambiguities of syntactic, logic and scmantic decoration (cf fig. 4). We have also defined three principles $(\$$ III.1) to order the questions if there is more than one to be asked. The first principle is: first of all, find out the right segmentation into simple sentences. The second principle is: for each common predicate in the MMC structure, find out the right subject, objects and adjuncts. The last principle is: for each simple sentence, find the right structure.

With those principles we are able to definc a strategy (cf fig. 5). We have also isolated some pattems in the three classes of ambiguity. The class of ambiguities of syntactic classes needs no refinement (\$ III.3.1). On the other hand we create four patterns of ambiguity of geometry ( $\$$ III.3.2) called: verbal coordination, argument structure of the verb, non verbal coordination, subordination; and three patterns of ambiguity of syntactic, logic and semantic decoration (§ III.3.3) called: logico-semantic labelling, argument order of direct transitive verbs, syntactic labelling.

Herc is an example with the interpretations for cacl pattern we have chosen:
Problem of clasi. Le pilote fermic la joite: The firmi pilot carries her. The pilot shuts the door.

Problem of verbal coordination. It regarde in photo ct la classe: He looks at the photograph and the class. He looks at the jhotograph and filcs it.

Problem of the argument struciure of the verb. II parle de l'école de cuisine: He talks abont the cooking school. He talks from the cooking school. He talks from the school about cooking.

Problem of non-yerbal coordination. Il prend des crayons et des cahiers noirs: He takes pencils and black notebooks. He takes black pcucils and black notebroks.

Problem of subordination. L'ecole de cuisine lyonnaise est fermec: The lyomaise cooking school is closed. The school of lyonnaise cooking is closed.

Problem of logico-semantic labelling. Picre fait porter des chocolats a Lucic: Pierre lets I.ucic carry chocolates. Pierre gets chocolates to be delivered to Lucic.

I'roblem of argument order of direct transitive verbs. Quel auteur cite ce conferencier: Which author this lecturer is quoting? Which lecturer this author is quoting?

Problem of syntactic labelling. Il parle de la tour Eiffel: He is talking about the Eiffel Tower. He is talking from the Eiffel Tower.

For each pattern we have defined a method to produce the appropriate dialogue (\$ II.3). These methorls use two kinds of processing; projection and paraphrase. Io build paraphrases we use basically threc operators: an operator of semantic replacement of occurrence, an operator of permulation of groups of occurrences and an operator of distribution of occurrences. 'The examples ( $\$$ IV) give an idea.

In conclusion we can say that our method is quite simple but fixed.once and for all. We are going to study two points in the noar future. The first one is to reduce the number of analysis and thus, by getting information from the user, reduce the time to spend on the disambiguation. The second is to try to build tools which will allow the linguist, designer of the linguistic part of the LIDIA system, to define its own methokls of disambiguation.

\section{Keywords}

Computer Aided Translation, Personal MT, Interactive Disambiguation, Dialogue Production 


\title{
Une solution au problème de la désambiguïsation interactive
}

\author{
Hervé BLANCHON
}

\author{
GETA, Institut IMAG (UJF \& CNRS) \\ BP 53X, 38041 Grenoble Cedex, France \\ e-mail : blanchon@imag.fr
}

\section{Résumé}

Dans le cadre du projet de TAO personnelle LIDIA $[1,3,4]$, un dialogue entre le système et le rédacteur permet d'obtenir une traduction de qualité. Ce dialogue a deux objectifs : standardiser et désambiguiser le texte. La standardisation concerne l'orthographe, le vocabulaire, les formules figees et quelques mesures stylistiques. La clarification concerne toutes les ambiguités que l'on peut rencontrer lorsque l'on fait de la traduction automatique.

Ici, nous ne nous intéressons qu'à une partie des traitements de désambiguïsation. Nous ne considérons que les problèmes repérables lors de l'examen de la structure MMC produite par l'analyseur, ambiguinte de classe, ambiguíté de géométric et ambiguïté de décoration syntaxique, logique et sémantique.

Nous examinons le type de structure d'où nous partons. Nous deffinissons alors les trois grandes classes d'ambiguiltés que nous proposons. Nous décrivons ensuite notre méthode en foumissant trois principes, une stratégie ot les traitements qui nous permettent, en raffinant les classes d'ambiguittés, d'obtenir finalement le dialogue. Cette méthode sera illustrée sur quelques exemples.

\section{Mots Clé}

Traduction Automatique, TAO personnelle, Désambiguïsation Interactive, Production de Dialogue

\section{La structure MMC}

L'analyseur utilisé dans le cadre du projet est un analyseur Multisolution et la structure qu'il produit a deux caractéristiques : elle est Multiniveau et Concrète.

\section{Multisolution}

La structure produite est dite "multisolution" car, pour chaque phrase, on produit toutes les analyses vérifiant le modèle syntagmatique, syntaxique et logicosémantique des grammaires utilisées. En effet, comme on veut obtenir l'analyse qui correspond aux intentions de l'auteur, il nous faut produire toutes les analyses vérifiant le modèle pour que le rédacteur aide le système à choisir la bonne.

\section{Multiniveau}

La structure produite est dite "multiniveau" car les nouds portent des décorations complexes qui représentent divers niveaux d'interprétation : le niveau des classes syntaxiques et des classes syntagmatiques, le niveau des fonctions syntaxiques et enfin le niveau des relations logiques et sćmantiques.

Pour préciser les choses, voici une brève description de ces divers niveaux d'interprétation.

- Le niveau des classes syntaxiques (classes terminales telles que : "NOM COMMUN", "ADJECTIF", "PREPOSITION", etc.) et des classes syntagmatiques (classes non terminales telles que : "GROUPE NOMINAL", "PHRASE INFINITIVE", "PHRASE VERBALE", etc.) donne le parenthésage de la phrase en groupes syntagmatiques. Les feuilles de l'arbre portent les classes syntaxiques et les nouds non terminaux portent les classes syntagmatiques.

- Le niveau des fonctions syntaxiques donne le rôle syntaxique de chaque nceud dans le groupe auquel il est attaché directement ("GOUVERNEUR" ou "TETE", "REGISSEUR", "SUJET", etc.). Avec ce niveau, on peut très simplement transformer l'arborescence en une arborescence de "dépendance syntaxique".

- Le niveau des relations logiques et sémantiques peut être considéré comme celui de la construction logique de la phrase, mettant en évidence les places des arguments attachés aux unités lexicales prédicatives et donnant l'interprétation sémantique des compléments et circonstants ("DETERMINATION", "PREMIER ARGUMENT D'UNE UNITE LEXICALE PREDICATIVE”, "CIRCONSTANCIEL INSTRUMENTAL", etc.).

Voici l'arbre d'analyse de la phrase "l'éleve calcule cette intégrale par la méthode des résidus".

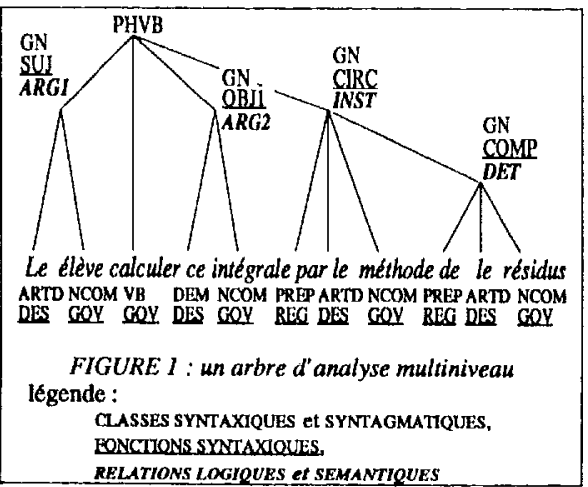




\section{Concrète}

La structure produite est dite "concrète", car on retrouve directement le texte analysé (en italique dans la figure 1) via le mot des feuilles de l'arbre. Cette propriété permet d'avoir un processus de génération des questions plus simple à mettre en oeuvre.

Pour produire le dialogue à partir de la structure MMC, nous sommes d'abord amenés à definir les types d'ambiguïté qui apparaissent dans la structure.

\section{Les ambiguités dans la structure MMC}

Nous considérons trois types d'ambiguïte, que nous traiterons differemment.

\section{Ambiguïté de classe}

Il y a ambiguïté de classe si une même occurrence est étiquetée par deux classes morphro-syntaxiques differentes dans deux arbres d'analyse distincts.

Le schéma suivant (figure 2) montre les deux arbres produits par l'analyse de la phrase "Devant cette somme, il hésite" dans laquelle 'devant' peut ctre interprété comme gérondif du verbe devoir ou bien comme préposition.

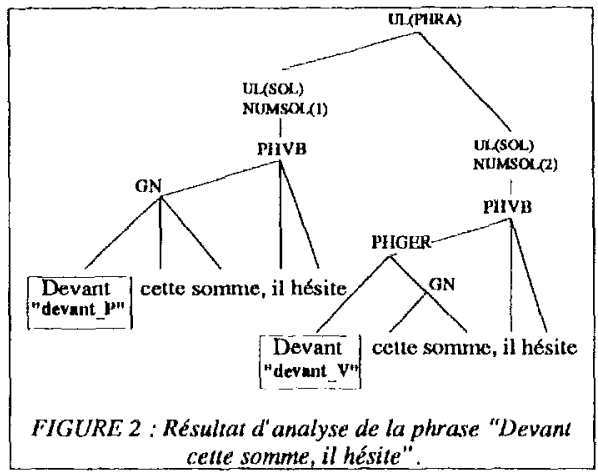

\section{Ambiguïté de géométrie}

Il y a ambiguitê de géometrie si deux arbres d'analyse différents ont des graphes distincts.
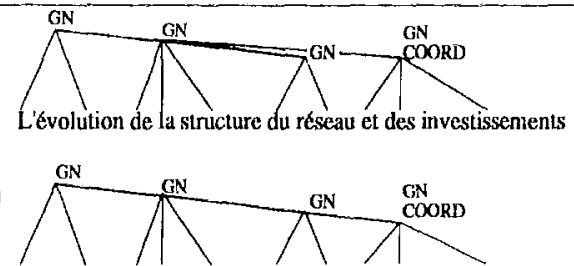

L'évolution de la stnucture du réscau et des investissements

FIGURE 3 : Résultat d' analyse du GN " $L$ 'évolution de la structure du réseau et des investissements".
La figure 3 montre les deux arbres produits pour lc groupe "L'évolution de la structure du réseau et des investissements" où l'on peut comprendre l'évolution de la structure du réseau et l'ćvolution des investissements (NUMSOL(1)) ou bièn l'evolution de la structure du réscau et l'évolution de la structure des investissements (NUMSOL(2)).

\section{Ambiguïté de décoration syntaxique, logique et sémantique}

Il y a ambiguïté de décoration syntaxique, logique et sémantique si pour des arbres ayant tous les mêmes groupes simples ${ }^{1}$, l'étiquetage des nouds non terminawx en fonction syntaxique et/on en relation logique et sćmantique est different.

La figure 4 montre les deux arbres produits par l'analyse de la phrase "Je vous parle de la tour Eiffel" où l'on peut comprendre qu'on hous parle depuis la lour Eiffel (NUMSOL(2)) ou qu'on nous parle au sujet de la tour Eiffel (NUMSOL(1)).

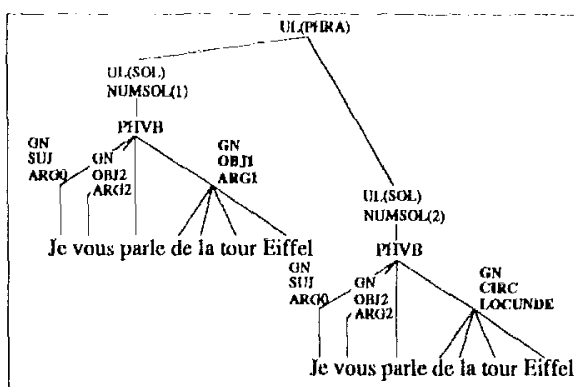

FIGURL' 4 : Résultal d" analyse de la phrase "Je vous parle de la Tour Eiffel".

\section{Méthodologie}

\section{Principes}

Nous avons défini trois principes qui fondent la stratégie que nous allons décrire plas bas. Les voici, par ordre d'importance :

1) trunver d'abord les bons groupes simples

2) trouver ensuite le sujet, les arguments et les circonstants de tous les prédicats

3) trouver enfin la structure du sujet, des arguments et des circonstants des prédicats

Ces trois principes ont été guidés par des raisons pragmatiques. Nous voulons en effet, et avant toute autre chose, trouver le bor découpage en groupes simples de

1 Un groupe simple est constitué de tout gouverneur accompagné de sa complémentation à gauche. Exemple "le chien", "le tout petit chien". Un gouverneur est un lexène qui n'est pas un mot outil. Exemple : "le" et "tout" sont des mots outil. 
la phrase. Ceux-ci forment, en effet, les ćléments de base qui permettent de produire le sens. Nous voulons ensuite choisir la construction de la phrase au plus haut niveau, c'est-à-dire la construction des prédicats. C'est finalement, lorsque l'on a construit correctement les prédicats que l'on peut s'intéresser à la structure interne des eléments qui les composent.

\section{Stratégie}

Comme plusieurs propriétés peuvent apparaître dans un résultat d'analyse, il faut lever les ambigüttés dans un ordre qui respecte les trois principes que nous avons poses.

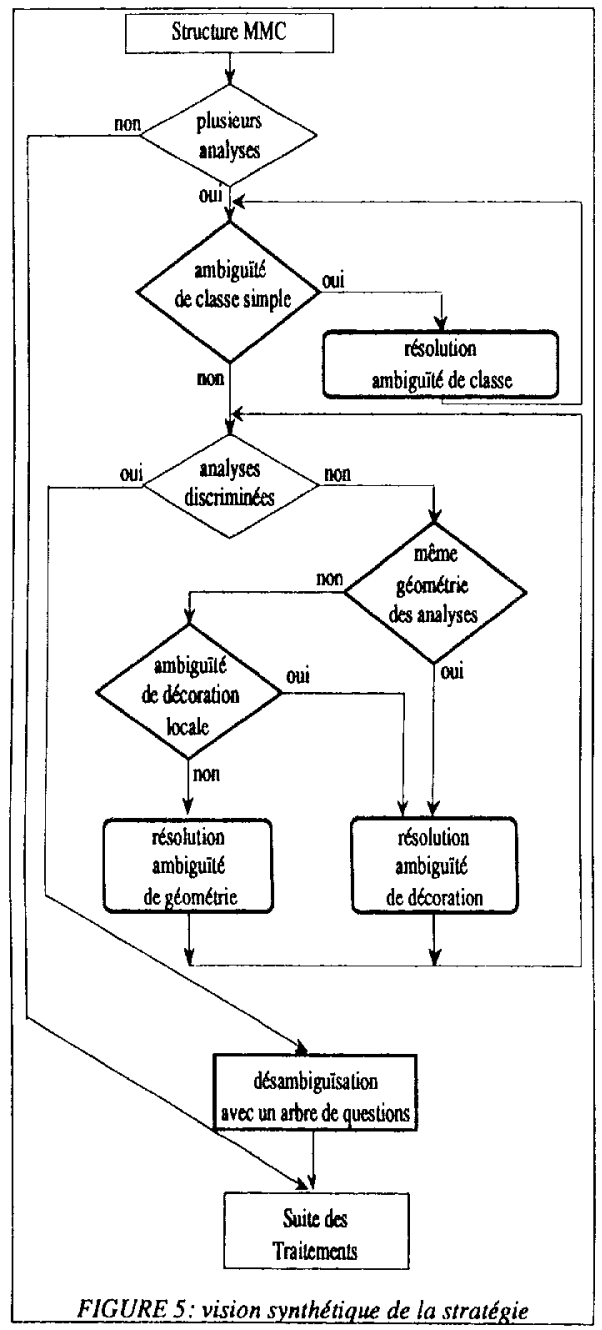

Les groupes simples ctant pour nous les briques de la phrase, il est necessaire de les obtenir le plus rapidement possible. $\mathrm{Si}$, pour toutes les occurrences d'une phrase, les classes syntaxiques associécs à chacune d'elles dans les diverses solutions d'analyse produites sont les mêmes, alors ces solutions sont composces des mêmes groupes simples. Si les classes sont différentes, les occurrences qui présentent cette propriété appartiennent ou non à un groupe coordonné de la phrase. Si elles n'appartiennent pas à un groupe coordonné, nous résoudrons une ambigunté de classe. Nous appellerons ce cas ambigulte de classe simple. Si elles appartiennent à un groupe coordonné, nous résoudrons le problème par une autuc méthode. Nous appellerons ce demier cas ambiguitte de classe avec occurrences coordonnées.

Lorsqu'on dispose des bons groupes simples, on peut chercher à construire le sujet, les objets et les circonstants de tous les prédicats. Pour ce faire, on localise la définition de l'ambiguïté de décoration en imposant que la propriété soit vérifiée pour une PHrase VerBale (PHVB) et non vérifiée par les groupes qui lui sont coordonnés. Cette ambigü̈té sera appelée ambiguîté de décoration locale.

Nous allons maintenant itérer une série de tests et d'actions jusqu'à produire un arbre de questions permettant de désambiguiser totalement la phrase. Chaque action a pour rôle de produire une partition de l'ensemble des arbres qu'elle reçoit et une question qui permet de choisir la bonne parmi les classes créées. Chaque classe ainsi créce est une nouvelle donnée de l'itération qui fabriquera si nécessaire une nouvelle question et de nouvelles classes. L'arbre de questions sera totalement produit lorsque la partition de l'ensemble des analyses initialement produites ne comportera que des classes à un seul elément.

La stratégie de production de l'arbre de questions est résumé dans la figure 5 .

\section{Traitement}

Nous allons examiner les trois types d'ambiguitte que nous avons definis et montrer comment on produit le dialogue pour chacun d'cux. Nous ne différencions pas ici l'ambiguîté de décoration et l'ambiguïté de décoration locale.

\subsection{Ambiguïté de classe}

Pour résoudre une ambiguïté de classe, on projette la partie de la phrase pour laquelle les classes diffèrent selon deux composantes, l'occurrence et la classe qui lui est associée. On pourra aussi proposer un dialogue plus détaillé.

\subsection{Ambiguïté de géométrie}

Pour résoudre une ambiguité de géométrie en respectant le second et le troisième principe, nous distinguons hiérarchiquement les problèmes suivants :

I. coordination verbale : problème de coordination pour lequel un même groupe peut être une PHrase VerBale coordonné ou non. C'est le cas d'ambiguñté de 
classe avec occurrences coordonnces que nous avons écarté tout à l'heure. Pour résoudre un tel problème, nous faisons un paraphrasage, par permutation, de chaque analyse qui met en valeur tout ce qui a pu être élidé, car nous disposons d'un modèle unique de phrase pour ce cas.

II. structure argumentaire du verbe : le verbe peut être complémenté de différentes façons. Par exemple, il peut être doté d'un sujet et d'un circonstant, ou bien, d'un sujet et d'un objet, ou enfin, d'un sujet, d'un objet, et d'un circonstant. Ici on projette la partic de la phrase qui permet de distinguer les différentes possibilités en remplaçant la préposition ambiguè qui introduit les complements par des prêpositions non ambiguds.

III. coordination non verbale : problème de coordination qui n'est pas lié à un problème de classe, le groupe coordonné n'est jamais une PHVB. Ici, on distribue ce qui peut l'être pour retrouver les interprétations sous une forme complètement explicite.

IV. subordination : un groupe établit différents liens de subordination avec les groupes qui le précedent. Par exemple, il peut être subordonné au groupe qui lui est contigu immédiatement à gauche ou à un groupe non contigu qui le précède. Dans ce cas, on rapproche par permutation les groupes subordonnés qui ont ćté séparés par un groupe intermédiaire. On se sert de parenthèses pour bien montrer les inots que l'on a regroupés.

\subsection{Ambiguité de décoration}

Résoudre une ambiguïté de decoration ou une ambiguîté de décoration locale nécessite les mèmes traitements. Dans le premier cas, on travaille sur un arbre tout entier, alors que dans l'autre on travaille sur un sous-arbre. On distingue les problèmes suivants :

I. étiquetage logico-sémantique : scules les relations logiques et sémantiques different entre les analyses. Il suffit de remplacer la préposition introduisant le groupe à problème par les prépositions non ambigues qui représentent chacunc des relations possibles.

II. ordre des arguments d'un verbe transitif direct : à une permutation près, tous les arbres ont le même étiquetage. On reconstruit par permutation toutes les interprétations selon un ordre "standard" sous une forme normalisce. On ne conserve que le gouverneur de chaque groupe sujet ou objet, ct on le munit du bon article avant de faire les projections dans l'ordre sujet + verbe + objet $1+$ objet 2 .

III. étiquetage syntaxique : il y a ambiguïté sur le rôle d'un groupe subordonné au verbe ou a un autre groupe simple. Il suffit de projeter la phrase en remplaçant la préposition ambigué introduisant le groupe qui pose problème par des prépositions ou des groupes sémantiquement équivalents non ambigus.

Les informations nécessaires au remplacement d'une occurrence se trouvent dans la base lexicale.

\section{Exemples}

Voici deux exemples de dialogues que l'on peut produire en utilisant la méthode décrite ci-dessus.

\section{La coordination verbale}

Phrase : Il observe la photo et la classe.

Arbres :

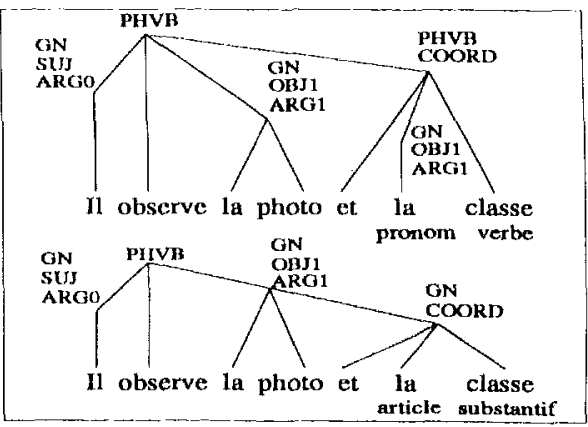

Dialogue : ambigüté

I'analyseur a rencontré un probleme de classes pour la phrase: Il observe la photo et la classe.

Que faut-il comprendre?

- il observe la classe

o il classe la photo

\section{L'étiquetage logico-sémantique}

Phrase : Pierre fait porter des chocolats à Lucie.

Arbres :

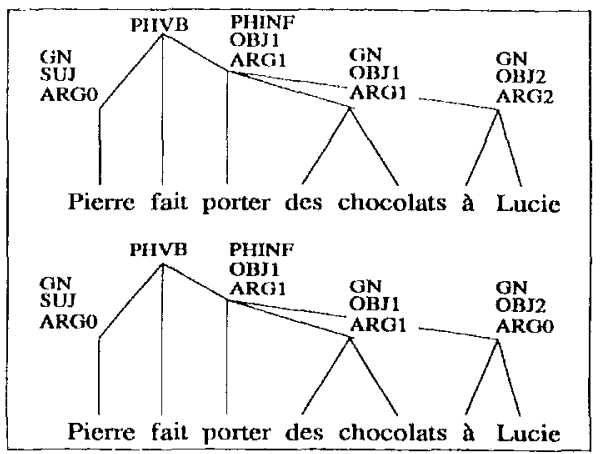


Dialogue :

\begin{tabular}{l}
\hline L'endyeur a rencontre un problume do ducorallon pour le phrme : \\
Pierre fait porter des chocolats a Lucie. \\
Que faut-ll comprendre? \\
o Pierre fait porter des chocolats pour Lucie. \\
O Pierne fait porter des chocolats par Lucie.
\end{tabular}

\section{La coordination non verbale}

Phrase : l'évolution de la structure du réseau et des investissements est éundiéce

Arbre : cf figure 3

Dialogue : mbiguité

L'analyzeur a rencontré un probleme de coordination pour la phrase : L'évolution de la structure du réseau et des investissements est étudiée.

Que faut.ll comprendre?

- L'évolution de la structure du réseau et l'évolution des investissements

- L'évolution de la structure du réseau et l'évolution de la structure des investissements

\section{Conclusion}

Vis à vis de la structure MMC que nous utilisons, une phrase est désambiguïsée lorsqu'on a trouvé les bons groupes simples, le bon rattachement de ceux-ci, les bonnes qualités de ces rattachements. La méthode que nous proposons traite tous ces problèmes. Pour trouver les bons groupes simples, il suffit de trouver la classe syntaxique de chaque occurrence. Pour trouver le bon rattachement des groupes simples, il faut gérer les problèmes de subordination et de coordination. Nous avons pris en compte le cas où ces problèmes influencent la construction des prédicats, ici intervient la qualité des rattachements. Enfin, nous résolvons le cas où se pose uniquement un problème de qualité des rattachements en differenciant les trois types de problèmes qui peuvent se poser.

Afin de diminuer le nombre des analyses produites et de réduire ainsi le temps à accorder au processus de désambiguîsation, nous réfléchissons aux moyens dont pourrait disposer l'utilisateur pour fournir au système des informations sur ce qu'il vient de rédiger. Ces informations devraient permettre de restreindre le modele syntagmatique et/ou syntaxique eV/ou logicosémantique de l'analyseur utilisé.
La méthode actuelle est figể. Nous désirons aller vers plus de généricité en offrant au linguiste un environnement avec lequel il puisse définir d'autres processus de désambiguisation. Cela veut dire qu'il faut des outils qui permettent de définir, de façon explicite on non, des types d'ambiguitté, des principes, une statégie et des méthodes de production du dialogue, comme nous l'avons fait ici. Dans notre approche, les principes sont implicitement pris en compte dans ta stratégie et dans les traitements. On peut par exemple imaginer que le linguiste décide de faire appel à des processus de génération qu'il aura développés sur le serveur de traduction.

\section{Remerciements}

Je tiens a remercier Jean-Philippe Guilbaud pour son assistance linguistique. Je remercie Egalement Christian Boitet, Mathieu Lafourcade, Kiki Levenbach, Gilles Sérasset qui ont corrigé ce document dans son fond et dans sa forme ; je suis bien sûr seul responsable des éventuelles imperfections qui subsisteraient.

\section{Bibliographie}

[1] Blanchon, H., (1990). LIDIA-I : Un prototype de TAO personnelle pour rédacteur unilingue. Proc. Avignon-90 : Le traitement du language naturel et ses applications. Avignon. 28 mai-1 juin, 1990.

[2] Blanchon, H., (1992). Désambiguisation interactive en TAO personnelle : poser les bonnes questions! . Proc. Avignon-92: Le traitement du language naturel et ses applications. Avignon. 3-5 juin, 1992.

[3] Boitet, C., (1989). Motivation and Architecture of the Lidia Project. Proc. MTS-89. Munich. 16-18 aotit, 1989.

[4] Boitet, C., (1990). Towards Personnal MT : on some aspects of the LIDIA project. Proc. Coling-90. Helsinki. 20-25 aôt, 1990. vol. 3/3 : pp. 30-35.

[5] Brown, R. D. \& Nirenburg, S., (1990). HumanComputer Interaction for Semantic Disambiguation. Proc. Coling 90 . Helinki. August 20-25, 1990. vol. 3/3 : pp. $42-47$.

[6] Chandler, B. \& Wood, M., (1988). Machine Translation For Monolinguals. Proc. Coling-88. Budapest. 1988 : pp. 760-763.

[7] Huang, X., (1990). A Machine Translation System for the Target Language Inexpert. Proc. Coling -90 . Helsinki. August 20-25, 1990. vol. 3/3 : pp. 364-367.

[8] Maruyama, H., Watanabe, H. \& Ogino, S., (1990). An interactive Japanese Parser for Machine Translation. Proc. Coling-90. Helsinki. August 20-25, 1990. vol. $2 / 3$ : pp. $257-262$.

[9] Melby, A. K., (1982). Multi-Level Translation Aids in a Distributed System. Proc. Coling-82. Prague. July 510, 1982. vol. 1/1: pp. 215-220.

[10] Wehrli, E., (1990). STS : An Experimental Sentence Translation System. Proc. Coling-90. Helsinki. August 20-25, 1990. vol. 1/3 : pp. 76-78.

[11] Zajac, R., (1988). Interactive Translation : a new approach. Proc. Coling-88. Budapest. August 22-27, 1988. 